\title{
Lessons from ten years searching the Congenital Rubella Syndrome (CRS) cases in young population of a developing country: Tehran; Iran.
}

Mohammad Vafaee-Shahi

Iran University of Medical Sciences

Samileh Noorbakhsh ( $\nabla$ samileh_noorbakhsh@yahoo.com )

Iran University of Medical Sciences https://orcid.org/0000-0002-1656-2388

Leila Tahernia

Tehran University of Medical Sciences

Sarvenaz Ashouri

Iran University of Medical Sciences

Aina Riahi

Iran University of Medical Sciences

Research

Keywords: Congenital Rubella Syndrome (CRS), Rubella

Posted Date: January 8th, 2021

DOI: https://doi.org/10.21203/rs.3.rs-64908/v1

License: (c) (1) This work is licensed under a Creative Commons Attribution 4.0 International License. Read Full License 


\section{Abstract}

Background: Maternal infection with rubella in the first trimester of pregnancy can result in developmental abnormalities in fetus such as hearing loss, heart abnormalities, impaired vision, and mental retardation.

Methods: This Multi-stage prospective/cross sectional study was carried out in three stages in three educational hospitals in Tehran (Rasoul akram, Akbar Abadi and Firoozabadi), during 2011-2012: In first stage, 186 infants at birth day. Rubella antibodies (IgG\&lgM) in cord blood was evaluated by Eliza method. In second stage, 89 infants suspected INTRA UTERINE INFECTION and in third stage 165 infants with SNHL were selected. Age matched control group were selected and examined concurrently. Rubella antibodies (IgG\&IgM) compared between cases and healthy matched infants.

Results: In first stage, significant correlation was found between vaccination history and rubella immunization of mothers ( $P$ value $=0.001)$. None of those with a history of vaccination had positive IgM. In second stage, confirmed CRS (positive IgM) was found in $4.4 \%$ of total CRS suspected cases (5/89). The positive RT-PCR detected in $1.5 \%$ of total CRS suspected cases (1/89). Except a positive correlation between abnormal neurologic findings and positive IgM in Confirmed CRS cases, other clinical findings were not related to serologic tests. In third stage, the highest frequency for Positive Rubella-lgM observed in the SNHL cases $<1$ year old (P-value $=0.14$ ). Rubella-IgG was significantly higher in age group $<1$ years $(P$-value $=0.001)$. There was no significant difference for Rubella-lgM $(P$-value $=0.6)$ between SNHL cases and controls but a significant $(P$-value $=0.000)$ difference was observed for Rubella-lgG which was more frequent in control group.

Conclusion: Active CRS surveillance is recommended strongly even in country with full rubella vaccination. Without active CRS surveillance;CRS especially, mild infection (not classic form) such as IUGR(intrauterine growth retardation), hearing loss, heart abnormalities, impaired vision, and mental retardation even in developed country might be missed. The necessity to add a booster vaccine dose to the national vaccination program 8 years after the last vaccination should be evaluated if needed. In addition, follow-up of safety status and studies to evaluate the success of the program may be necessary and is recommended.

\section{Introduction}

The congenital infections, referred to as "INTRA UTERINE INFECTION ", are of great importance in every country in terms of pre-natal and postnatal controls (1). The most severe form of congenital intrauterine infections is congenital rubella. The diagnosis of CRS (congenital rubella syndrome) is considered with the classic clinical triad of cataracts, heart disease and hearing loss; although, many neonates have only one symptom at presentation or will progress symptoms during infancy (2). Maternal infection with rubella in the first trimester of pregnancy can result in developmental abnormalities in fetus such as hearing loss, heart abnormalities, impaired vision, and mental retardation (2). About $50 \%$ of symptomatic 
newborns with congenital rubella have hearing loss which may end in cochlear implantation. According to a study in Brazil, the health care cost of a congenital rubella case in the first year of follow-up was US $\$$ 61,824 , that will continue for the following years until the end of life (3). Infants who are exposed to this agent during the second and third trimesters of pregnancy are often born with mild rubella infection. But in $20-30 \%$ of newborns, hearing disorders will be diagnosed later which could be bilateral or asymmetrical, ranging from mild to severe (4). The incidence of CRS is mostly related to the time of rubella epidemic occurrence. The incidence of congenital rubella per 1,000 live births was reported in some countries in different years including: Israel 7.1 (1972), Oman 0.7 (1993), Panama 2.2(1986), Singapore 5.1 (1969) 5.1 and Sri Lanka 5.5 (1994) (5). Given the teratogenic potential of rubella infection, efforts were made to screen and test a vaccine that eventually led to the production of a weakened live rubella vaccine in 1969. The widespread vaccination of all children as well as young adults, especially girls had such a significant impact on incidence of congenital rubella in the United States (6).

According to the WHO recommendation in countries where Rubella has not been eradicated, assessment of Rubella epidemics should be used to activate CRS surveillance. Routine CRS surveillance should be done according to the guideline of World Health Organization (5). However, some symptoms may be late onset. After one year the diagnosis would be very difficult. The number of CRS cases per 1000 live births should be reported annually. Active detection of cases of congenital rubella is required even with a major symptom (according to WHO classification). With estimation of births, it could be possible to estimate the number of congenital rubella cases per 1000 live births (5). Before MR vaccination campaign in Iran (2003) rubella was not a reportable disease. First report for Rubella immunity in Iran (1974) was published by Saidi in WHO bulletin (5). The prevalence of CRS was reported in the vicinity of 0.2/1000 prior to rubella vaccination in Iran, the statistics suggest that rubella vaccination could potentially prevent about $12 \%$ of sensorineural hearing loss in Iranian children. (by Sadighi et al. 2005) (7). According to some other studies in Iran, congenital rubella was responsible for $12 \%$ of cases of sensory-neural hearing loss (SNHL).

The prevalence rates of rubella and CRS in Iran were not known; therefore, the risk of exposure in pregnant women was not clear. So in last decades many Iranian authors tried to determine the risk of CRS in our country (7). Now, due to limited disease surveillance and reporting systems, data on the incidence of rubella and CRS in Iran are scant. This study provides data on the prevalence of CRS in three hospitals located in Tehran, Capital of Iran.

\section{Methods}

This Multi-stage prospective/cross sectional study was carried out in three stages: All project partners adheres to the principles of the Helsinki Declaration. Written informed consent was obtained from the parents of all children enrolled in the study. Performing all clinical examinations and diagnostic tests will be at the expense of the plan. 
In all three stages, sampling was done with available samples (by easy method). Total cases were selected patients based on criteria for suspected congenital rubella set by the World Health Organization (CRS-suspected). The questionnaires were filled out for patients selected based on description, clinical examination and course of disease with primary suspicion of congenital rubella. Data included patient characteristics, name, sex, season, type of major and minor disease, and maternal age at pregnancy. The information on infants, antibody levels in their cord blood and seropositivity was collected in a checklist containing study variables.

In first stage of perspective/ cross-sectional study, total 186 infants were selected by simple sampling in three educational hospitals in Tehran (Rasoul akram, Akbar Abadi and Firoozabadi) during 2011-2012. ( Figure-1)

In second stage of a cross-sectional, case - control study, total 163 blood samples of infants with suspected INTRA UTERINE INFECTION were compared with a group of healthy matched infants. The preliminary tests were requested after initial examinations by a paediatrician. Diseases such as chromosomal and metabolic diseases, liver, kidney and bone disorders, etc. were diagnosed in 33 patients and all were excluded from this study. Finally, 89 infants were eligible for INTRA UTERINE INFECTION screening. The Patients were divided into 3 groups of suspected, compatible or confirmed CRS and then data were entered into SPSS software for description and analysis. Then frequency of seropositivity in these antibodies was reported for these patients.

In third stage of a cross-sectional / case - control study, Rubella serology compared between $95 \mathrm{SNHL}$ (Sensory-neural hearing loss) cases and 70 controls (healthy children). SNHL is when the hearing loss is greater than $30 \mathrm{db}$ at 3 consecutive frequencies in PTA. The type of hearing loss (unilateral or bilateral) was determined and the severity of hearing loss in the patient group was classified according to the following classification: In borderline hearing loss ((Borderline: $16-30 \mathrm{db})$, they may not hear $10 \%$ of the words in crowded room. In mild hearing loss (Mild) 30-50db, they only hear some words. They are often considered relatively deaf. In moderate hearing loss (Moderate: 40-55db), most words are not heard in normal conversation. Around 2 mililiters of blood sample was taken from patients and centrifuged and stored at Rasool Akram Hospital Research Laboratory. In control group, 70 children were selected in the same age group who were referred to the outpatient clinic for examinations, or those who had undergone elective surgery in the surgical ward (without hearing impairment). The remain of blood was used for serology tests. In all stages, Rubella immunity (IgG\&lgM) in blood was evaluated by Elisa method. The samples centrifuged, transported and restored in -30 centigrade freezer in our Research Laboratory. All serum samples were tested by ELISA method (RADEM- Italy) using a cut-off point by the laboratory official and the results were interpreted. The serologic results were processed by Muli Skan EX and ELISA. Selected samples (positive Rubella IgM) were tested for rubella RNA by reverse transcriptase-polymerase chain reaction (RT-PCR)

Statistics: The mean and median indices and SD dispersion index were used to describe the data. Chi square test was also used to investigate the difference between seropositivity in preterm and term 
infants. Inclusion criteria were as follows: Age from birth to the end of one year, based on criteria set by the World Health Organization - suspected CRS (having at least one major symptom or 2 minor symptoms in favour of congenital rubella). Major symptoms included of Congenital heart disease, and/or suspicion of deafness, and/or one or more ophthalmic signs. Minor Symptoms: Jaundice on birth day of hepatomegaly, splenomegaly, steatosis, petechiae or purpura, muffin skin lesions, developmental disorders, seizures. Exclusion criteria included: no consent to participate in the study - evidence of other causes describing clinical symptoms.

\section{Results}

First stage: Specific rubella-IgM was positive in $4.2 \%$ and rubella-lgG was positive in $88.2 \%$ of neonates. There were no differences between IgG positive and IgG negative groups in mean age of mothers, mean gestational age and birth weight of infants. $87.5 \%$ of subjects without history of vaccination were IgG positive, while $62.2 \%$ with a history of vaccination were lgG positive. A significant correlation was found between vaccination history and rubella immunization ( $P$ value $=0.001)$. None of those with a history of vaccination had positive lgM.

Second stage: Total 89 blood samples of infants with suspected INTRA UTERINE INFECTION infection were compared with a group of healthy matched infants. $55.2 \%$ were male and $44.8 \%$ were female. Range of age was 1 to 12 months-old; mean age was $4.74+33.78$ months. Rubella-lgM and IgG found in $8.1 \%$ and $33.3 \%$ of infants with INTRA UTERINE INFECTION suspected cases $(n=89)$. Although, the positive Rubella-IgM in cases was 1.5 times more than healthy infants but it was not significant (P-value $=0.1$ ). In contrast, previous immunity in control group was significantly higher (80\%) than case group $(33 \%),(p=0.0000)$.Table-1

Overall $87 \%$ of 89 Children with INTRA UTERINE INFECTION suspected infants (having at least one major symptom or 2 minor symptoms in favour of CRS) were positive for anti-rubella IgG, negative IgM and CRS were not the final diagnosis in them. Rubella-IgM and IgG were positive in $8.1 \%$ and $33.3 \%$ of infants with INTRA UTERINE INFECTION suspected infants. The mean age in Rubella-IgM positive group was $5.41+44.49$ months compared to $4.21+\_3.69$ months in negative group which did not differ significantly $(P$-value $=0.4)$. The mean age in Rubella-lgG positive group was $4.2 \pm 4.12$ months compared to $4.9+_{-}$ 3.25 months in negative group which was not significant. (P-value $=0.6$ ). The age of mothers of infants with Rubella-IgM positive infection was 25-26 years. Previous immunity (positive Rubella-lgG) in control group was significantly higher than INTRA UTERINE INFECTION cases ( $80 \%$ vs $33 \% ; p=0.0000)$. Table- 1

Significant difference was observed in congenital heart disease and all clinical signs between groups with positive and negative Rubella IgM $(P=0.000)$.CRS diagnosed according to WHO criteria were diagnosed. The confirmed CRS (positive-IgM) was found in $4.4 \%$ of total CRS suspected cases $(5 / 89)$, In deed, positive RT-PCR detected in 1.5\% of total CRS suspected cases (1/89). Except a positive correlation between abnormal neurologic findings and positive IgM in Confirmed CRS cases, other clinical findings were not related to serologic tests. 
Although, the recent infection in cases was 1.5 times more than healthy infants but it was not significant. In contrast, previous immunity in control group was significantly higher $(80 \%)$ than case group $(33 \% ; p=$ 0.0000). No difference was seen in isolated signs (icter, hepatosplenomegaly, mental retardation, eye involvement, deafness, IUGR, CNS involvement) but significant difference was observed in congenital heart disease and all clinical signs between groups with positive and negative $\lg M(P=0.000)$.

Third stage: Rubella-IgM and IgG were positive in $5.3 \%$ and $17.2 \%$, of infants with SNHL, respectively. The recent infection (IgM) was not different between case and control groups but in contrast previous immunity in control group was significantly higher than case group $(p=0.000)$. Mean age of cases with positive Rubella-IgM cases was lower than negative ones (6.4 vs 37.6 months; $P=0.02)$. The mean age in the cases with positive Rubella-lgG was significantly higher than non-immune ones (49.8 versus 33 months; $P=0.04$ ). Although, the highest frequency for Positive Rubella-lgM observed in the age group; $<1$ year of age but without significantly different in various age groups. (P-value $=0.14)$.

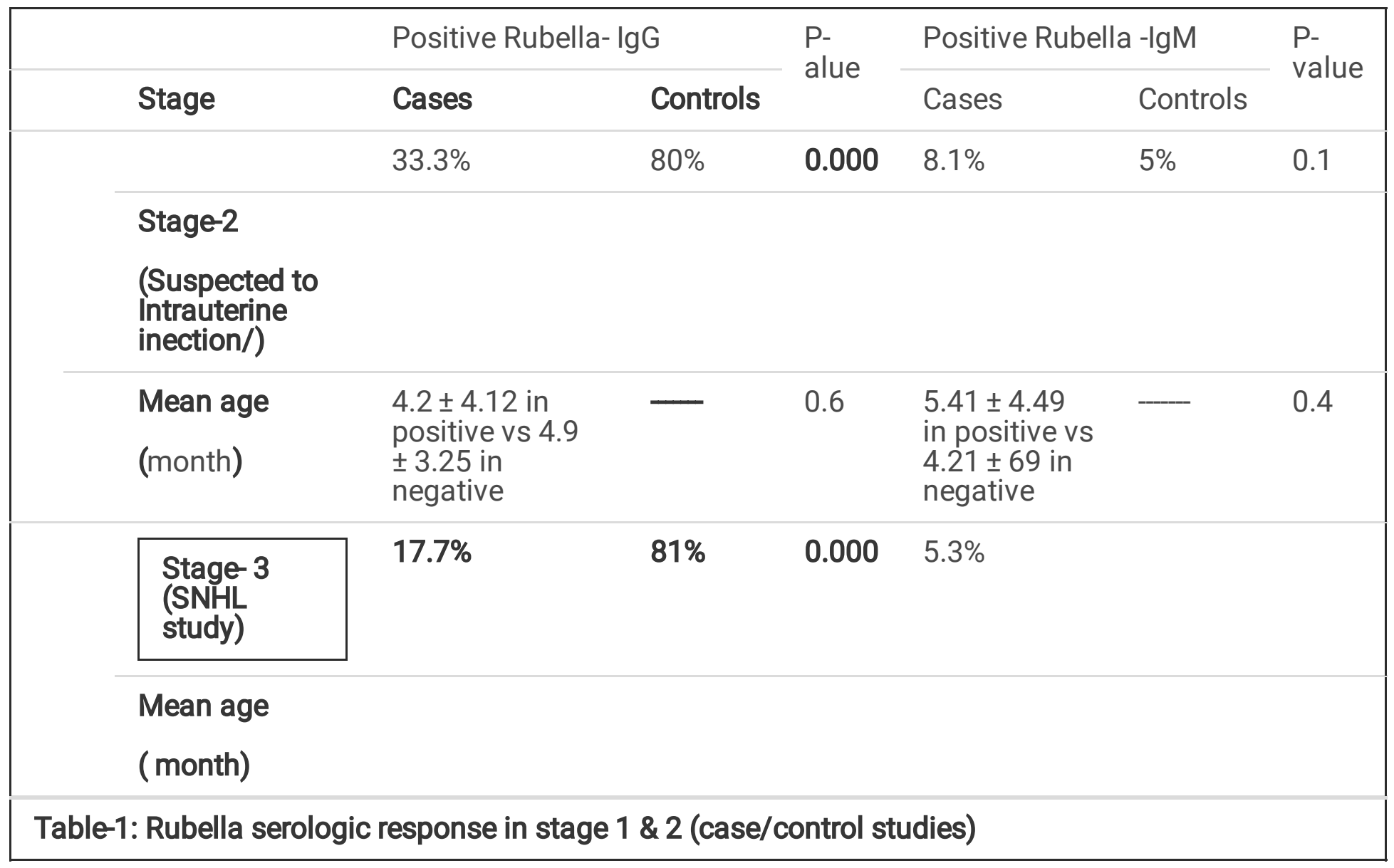

\section{Discussion}

Rubella specific IgM is detectable in six months after birth or even until one year of age. Its detection is more indicative of pre-natal rubella infection than postnatal infection. In $95 \%$ of CRS infants, specific rubella IgG can be acquired after 6 months of age (the age of maternal IgG eradication) (8). Positive IgG 
antibody after 6 months of age may indicate either prenatal or postnatal infection. The detection of IgG1 (which is low) is diagnosed as prenatal infection (8). The virus can be recovered from the posterior pharynx and conjunctiva, CSF, or urine. In the CSF, the virus may also appear for several years. There are two approaches to serologic diagnosis. Antibody against rubella can be measured in umbilical cord serum. If IgM is found because it is of embryonic origin, the diagnosis is definitive. However, incomplete deletion of IgG (most of which are of maternal origin) or rheumatoid factor can give rise to false positive results. In the early days, the specific Ab titer against rubella may not be high enough to get positive. The second approach is to monitor whether IgG levels remain permanent or not. Maternal Ab has a half-life of 30 days, they decrease by half in one month and disappear in 6 to 12 months. Stable lgG levels at this age, especially at high levels, indicates intrauterine infection with rubella. Serum should be checked at 3 , 5 and 6 months and followed with another sample if necessary (9). The availability of specific tests for prenatal diagnosis can facilitate counselling for parents concerned about maternal contact or infection. The use of PCR has recently been suggested. Sampling and the maternal infection time affect test sensitivity, which is $100 \%$ in amniotic fluid samples and $83 \%$ in chorionic villus samples. Repeat tests can increase accuracy (10).

Some investigations in Iran showed that rubella immunity in women of childbearing age fluctuated between $70 \%$ and $95 \%$ from 1968 to 1995 . The rate of rubella immunity in this population in 1995 was estimated $80 \%$. Therefore, this year in the present study was considered a non-epidemic year. The number of children born with deafness due to rubella would be expected to increase in epidemic years (10).

The present study with the aim of investigating the safety of rubella in the blood of infants (maternal and neonatal immunity) 8 years after the national vaccination in 2011 showed that approximately $75 \%$ of infants were immunized and $25 \%$ were immune deficient and prone to rubella infection. This will increase the risk of congenital rubella syndrome. According to recent studies, after 8 years of this vaccination program, there has not been a detailed assessment of the maternal-fetal transmitted immunity via cord and placenta (11). In present study of 154 infants, $58.4 \%$ of mothers had a history of vaccination in or after national vaccination program in 2003. No IgG immunogenicity was detected in $27.3 \%$ of population. This might be due to less number of subjects in this study compared to similar studies.

The mean age in infants with previous immunity and recent infection and group with no immunity and no recent infection was about 4-5 months and was not different. Over time, acute phase IgM antibodies gradually decreased in neonates with congenital infection. Therefore, the elimination of this antibody was expected at the age of 5-6 months. Positive antibody before the age of one year,can be due to intrauterine infection or placental transmission from mother. It may be concluded that infants suspected to have intrauterine infection INTRA UTERINE INFECTION were more prone to intrauterine infection than healthy infants because of lack of maternal protective antibodies (12). Except for cardiac disease, other clinical signs were in favor of congenital rubella infection that showed a marked increase in the group of infections. This indicated the importance of having at least one other symptom (other than heart disease) as an adjunct to the diagnosis of congenital rubella syndrome in infants suspected of Intra uterine infection . 
The incidence of congenital rubella in every 100 infants suspected of Intra uterine infection was about $8.1 \%$ in our study. It was lower than incidence in Tanzania (12\%). There were no cases of congenital rubella in live born babies of healthy infants (13). In this study, the proportion of cases diagnosed with CRS at the beginning of infancy was similar to $34 \%$ in the UK under 6 months of age. It is estimated that the prevalence of bilateral hearing loss increases to 1.5-2/1000 children under the age of 6 years [14]. In Iran, the prevalence of hearing loss is $1 / 1000$ children [7]. Regarding the prevalence of SNHL and the estimated AR in this study (12\%), congenital rubella was considered as the cause of deafness in approximately 93 children aged 1-4 years of age in the present study. If we assume that the epidemiology of rubella in Tehran, the capital city of Iran, is similar to other areas of Iran, we obtain the estimate that in the year of the present study there were approximately 620 children (1 to 4 years) in Iran with deafness that could have been prevented by rubella vaccination. According to some studies in Iran, congenital rubella is responsible for $12 \%$ of cases of sensory neural hearing loss. Nine mothers ( $41 \%)$ of 22 deaf, seropositive children in the present study reported a history of rubella, rash, or rubella exposure during pregnancy. Other studies have also reported between $40 \%$ and $75 \%$ of deaf seropositive children had such a maternal history (14). In this study, the degree of hearing loss in children who attended deaf educational centres was often higher than $50 \mathrm{~dB}$ (severe to profound hearing loss), and their hearing loss was bilateral. Thus, children with low severity of deafness were not included in this study, and the relation between severity of deafness and congenital rubella could not be estimated. About $20 \%$ of the children in deaf educational centres were not included because of a history of MMR (Measles, Mumps and Rubella) vaccination, which may lead to an underestimation of the OR. Meanwhile, experience in other countries suggests that if MMR vaccination coverage is less than $60-70 \%$, it may actually increase the age of infection, and therefore the incidence of CRS (14).

All above finding indicate that the prevalence of CRS in Iran is approximately 0.2/1000 (Before rubella vaccination in Iran). According to research done in Iran (during the year of this study), the rate of rubella immunity has reached about $80 \%$ (15); however, this rate of immunity is similar to that in other countries during the rubella pandemic of the 1960's, which claimed thousands of victims. Epidemiological evidence had shown that while rubella virus was continuing to circulate among children, there was still a risk of infection in pregnant women, even though only $3 \%$ of them were non-immune, and there was little prospect of eliminating CRS (16). If we assume that the epidemiology of rubella in Tehran (capital city of Iran), is similar to other areas of Iran, we obtain the estimate that in the year of the present study there were approximately 620 children ( 1 to 4 years) in Iran with deafness that could have been prevented by rubella vaccination. he world has now accumulated 35 years of lessons on use of rubella vaccine, and some striking examples of how rubella vaccination strategies should and should not be applied (17). Most importantly, studies in developed countries had generated the following recommended vaccination program: routine MMR vaccination at 12-14 months of age followed by a second dose of MMR vaccine at 4-6 years (both sexes) (7). This study clearly showed the necessity for suitable rubella vaccination program in Iran. However, inadequately implemented childhood vaccination runs the risk of altering rubella transmission dynamics and can lead to increase insusceptibility in women of childbearing age 
with the potential of increased numbers of cases of CRS. Consequently, it is essential that childhood vaccination programs achieve and maintain high levels of coverage $(7,17)$.

\section{Conclusion}

The present findings indicated that the prevalence of CRS was approximately $0.2 / 1000$ before rubella vaccination in Iran; vulnerability to rubella infection had increased in the at risk population, which can increase CRS complications, undermine community health and impose additional costs on the country. All finding indicated that implementation of appropriate rubella vaccination programs could potentially prevent about $12 \%$ of cases of SNHL loss in Iranian children. An appropriate surveillance system is needed because the introduction of rubella vaccine without epidemiological data and an adequate monitoring program may result in the shifting of rubella cases to higher ages, and increased incidence of CRS. Therefore, it is suggested that in addition to pursuing similar studies and examining a larger volume of individuals to obtain more accurate results, the national vaccination program against rubella will continue to be pursued. Furthermore, more efforts are required to inform the public about the reasons for the necessity of a strict vaccination program. Also, identifying, training, and evaluating at risk individuals and recommendation of vaccination before pregnancy seems necessary. so it seems reasonable that maternal screening and immunization of women should be included in the prenatal screening program. Rubella vaccine should be provided in rural health centers and health homes. In addition, the need to reimplement the vaccination program for women and girls aged between 5 to 25 years should be considered in the agenda to ensure that the program is repeated more accurately and expeditiously (if necessary). The necessity to add a booster dose to the national vaccination program 8 years after the last vaccination should be evaluated if needed. In addition, follow-up of safety status and studies to evaluate the success of the program may be necessary and is recommended.

\section{Declarations}

\section{Ethics approval and consent to participate:}

This study was accredited by Ethical Committee of Iran University of Medical Sciences. Helsinki Declaration was respected across the study and the informed consent form was signed by the parents.

\section{Competing interests:}

The authors declare no conflict of interest in preparing this study.

\section{Funding:}

This study received no specific grant from any funding agency in the public, institutions or not-for-profit sectors.

All authors confirm Consent for publication 
All authors confirm Availability of data and materials

\section{Authors' contributions:}

NS designed and supervised the study, writing the initial report.

VM visited and interpreted the patients data.

TL were major contributors in rewriting and English editing the manuscript.

SA : Methodology and statistics

\section{Acknowledgment}

We thanks for support of the Infectious Diseases Research Center of Iran University of Medical Sciences

\section{References}

1. Julie KimStamos,Anne H.Rowley. Timely Diagnosis of Congenital Infections. Volume 41, Issue 5, October 1994, Pages 1017 1033.doi.org/10.1016/S0031-3955(16)38843-5.

2. Chauhan N, Sen MS, Jhanda S, Grover S. Psychiatric manifestations of congenital rubella syndrome: A case report and review of literature. J Pediatr Neurosci. 2016;11 (2):137-139. doi:10.4103/18171745.187643.

3. Babigumira JB, Morgan I, Levin A. Health economics of rubella: a systematic review to assess the value of rubella vaccination. BMC Public Health. 2013;13:406. Published 2013 Apr 29. doi:10.1186/1471-2458-13-406.

4. White SJ, Boldt KL, Holditch SJ, Poland GA, Jacobson RM. Measles, mumps, and rubella. Clin Obstet Gynecol. 2012;55(2):550-559. doi:10.1097/GRF.0b013e31824df256.

5. Vaccine Assessment and Monitoring Team of the Department ofVaccines and Biologicals Ordering code: WHO/V\&B/99.22 Printed: December1999 who.int/gpv-documents/World Health Organization. Vaccines and Biologicals. CH-1211 Geneva 27, Switzerland.

6. Hendriks J, Blume S. Measles vaccination before the measles-mumps-rubella vaccine. Am J Public Health. 2013;103(8):1393-1401. doi:10.2105/AJPH.2012.301075

7. Sadighi J, Eftekhar H, Mohammad K. Congenital rubella syndrome in Iran. BMC Infect Dis. 2005;5:44. Published 2005 Jun 6. doi:10.1186/1471-2334-5-44.

8. Carmen L. Charlton, Alberto Severini. Dilemmas and Pitfalls in Rubella Laboratory Diagnostics in Low Prevalence or Elimination Settings.Curr Treat Options Infect Dis (2016) 8:329-342. DOI 10.1007/s40506-016-0090-8.

9. Dimech W, Grangeot-Keros L, Vauloup-Fellous C. Standardization of Assays That Detect Anti-Rubella Virus IgG Antibodies. Clin Microbiol Rev. 2016;29(1):163-174. doi:10.1128/CMR.00045-15. 
10. VIDIGAL, Paula Vieira Teixeira et al . Prenatal toxoplasmosis diagnosis from amniotic fluid by PCR. Rev. Soc. Bras. Med. Trop.Uberaba ,v. 35, n. 1, p. 1-6, Feb. 2002.

11. Faucette AN, Pawlitz MD, Pei B, Yao F, Chen K. Immunization of pregnant women: Future of early infant protection. Hum Vaccin Immunother. 2015;11(11):2549-2555.

12. Patricia Palmeira, Camila Quinello,Ana Lú cia Silveira-Lessa, Cla' udia Augusta Zago, Magda Carneiro-Sampaio.IgG Placental Transfer in Healthy and Pathological Pregnancies. Hindawi Publishing Corporation Clinical and Developmental Immunology Volume 2012, Article ID 985646, 13 pages

13. Masresha B, Shibeshi M, Kaiser R, Luce R, Katsande R, Mihigo R. Congenital Rubella Syndrome in The African Region - Data from Sentinel Surveillance. J Immunol Sci. 2018;Suppl:146-150.

14. Marashi sm, Tabatabaei $\mathrm{H}$, Mahmoodi M, Nategh R, Mokhtari-Azad T. Prevalence of Rubella and HCMV Antibodies among Neonates. Iranian Journal of Virology 2011;5(3): 34-39 @ 2011, Iranian Society for Virology.

15. Soleimanjahi H1*, Fotouhi F1, Bamdad T1 Evaluation of Antibodies against Rubella Virus in a Mass Campaign Vaccination in Tehran, Iran .Iranian Journal of Virology 2010;4(3\&4): 7-11

16. Almassinokiani, S. Noorbakhsh, M. Rezaei, A. Almasi, H. Akbari, S. Asadolla, P. Rahimzadeh andM. Saberifard. What do we need to eradicate rubella in the Islamic Republic of Iran? Eastern Mediterranean Health Journal: EMHJ • Vol. 19 No. 9 • 2013: 807-11.

17. Report of a meeting on preventing congenital rubella syndrome: immunization strategies, surveillance needs. Geneva, 12-14 January 2000. The Department of Vaccines and Biologicals. World Health Organization Department of Vaccines and Biologicals CH-1211 Geneva 27, Switzerland. E-mail: vaccines@who.ch.

\section{Figures}




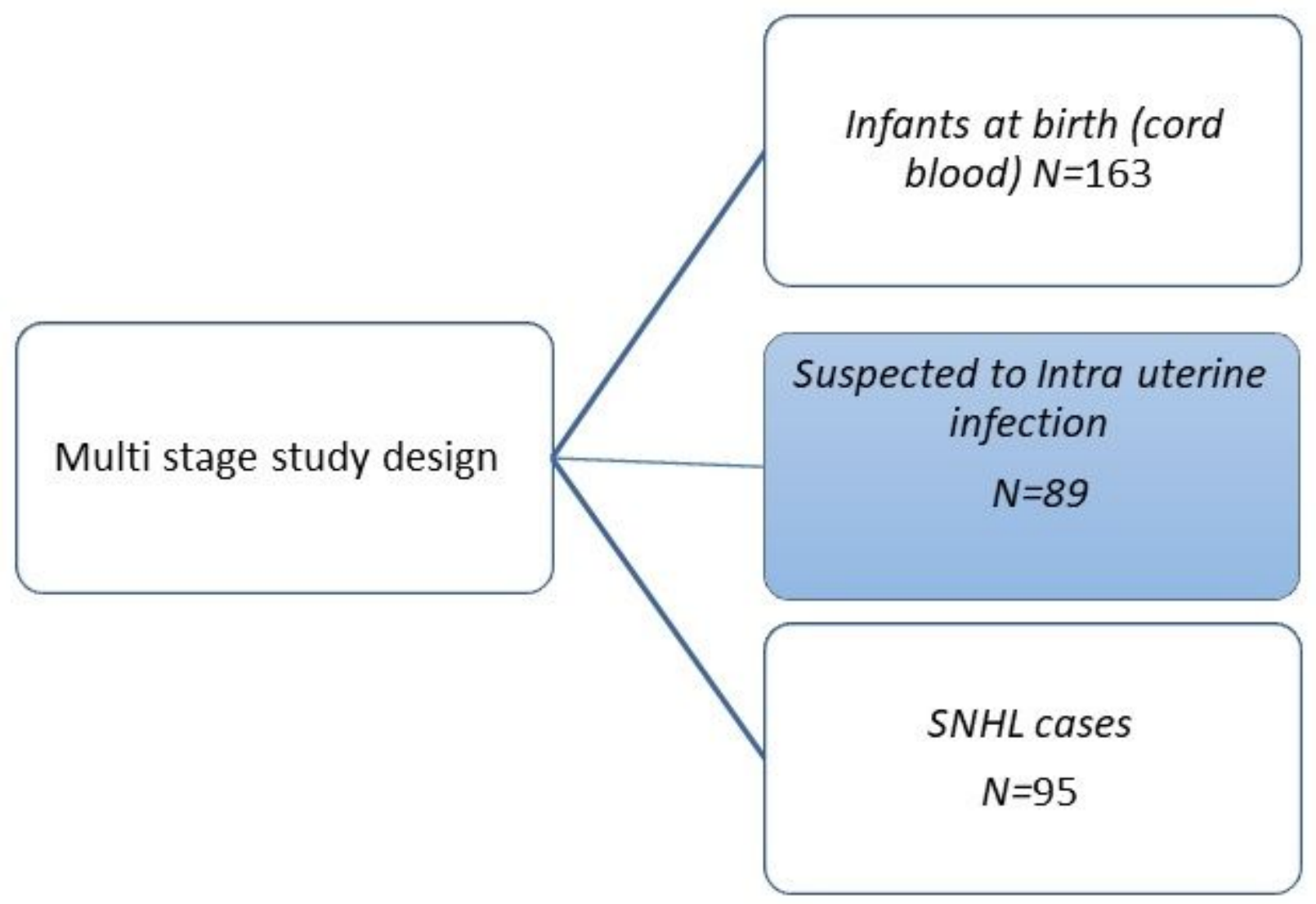

Figure 1

Multi-stage study design and selection the cases . 


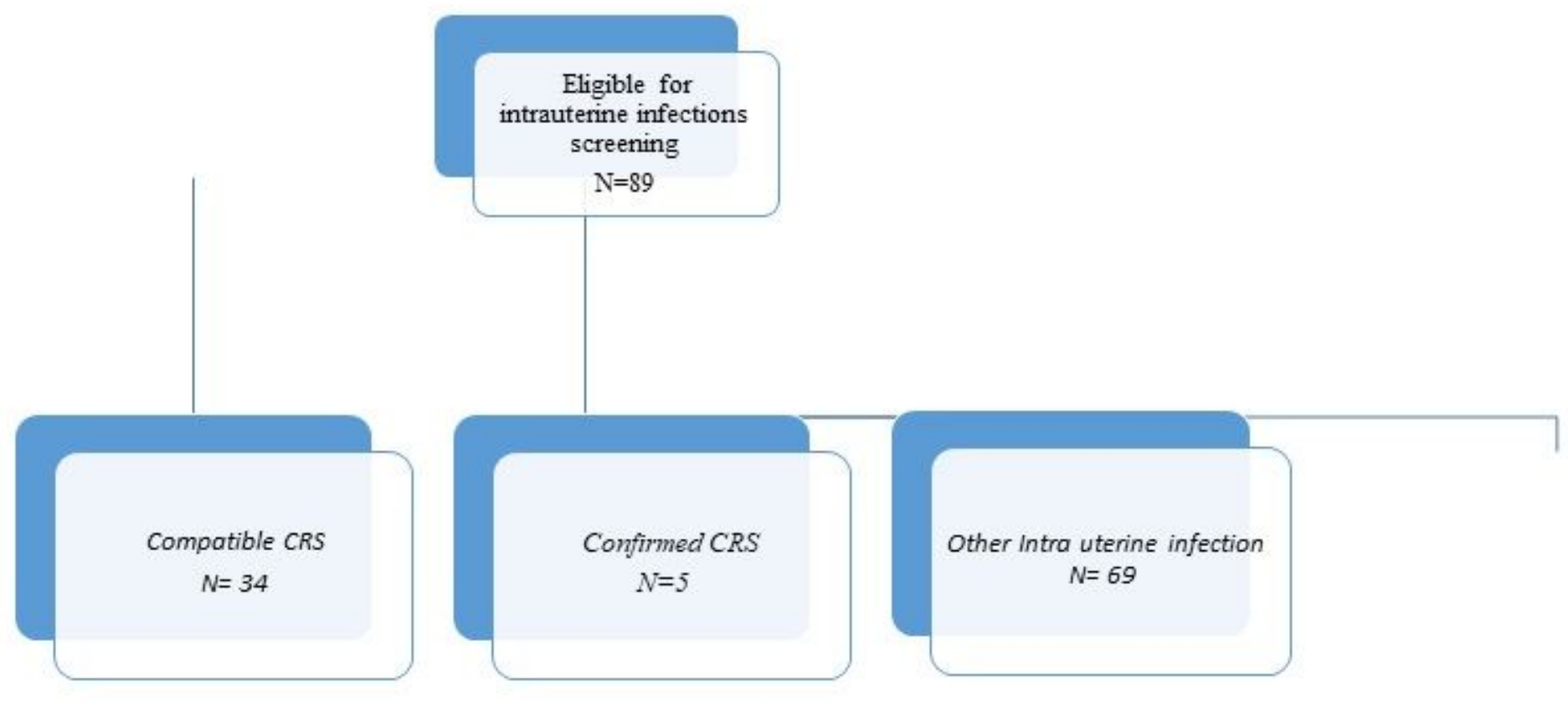

Figure 2

Final diagnosis in 89 infants were eligible for intrauterine infection screening 